\title{
Evaluation of objectivity, reliability and criterion validity of the Key Indicator Method for Manual Handling Operations (KIM-MHO), draft 2007
}

\author{
André Klußmann ${ }^{\mathrm{a}, \mathrm{b},}$, , Hansjürgen Gebhardt ${ }^{\mathrm{a}}$, Monika Rieger ${ }^{\mathrm{b}}$, Falk Liebers ${ }^{\mathrm{c}}$ and Ulf Steinberg ${ }^{\mathrm{c}}$ \\ a Institute of Occupational Health, Safety and Ergonomics (ASER), Corneliusstrasse 31, D-42329 Wuppertal, \\ Germany \\ ${ }^{\mathrm{b}}$ Institute of Occupational and Social Medicine, University Hospital of Tübingen, Wilhelmstrasse 27, D-72074 \\ Tübingen, Germany \\ ${ }^{\mathrm{c}}$ Federal Institute for Occupational Safety and Health (BAuA), Nöldnerstrasse 40-42, D-10317 Berlin, Germany
}

\begin{abstract}
Upper extremity musculoskeletal symptoms and disorders are common in the working population. The economic and social impact of such disorders is considerable. Long-time, dynamic repetitive exposure of the hand-arm system during manual handling operations (MHO) alone or in combination with static and postural effort are recognised as causes of musculoskeletal symptoms and disorders. The assessment of these manual work tasks is crucial to estimate health risks of exposed employees. For these work tasks, a new method for the assessment of the working conditions was developed and a validation study was performed. The results suggest satisfying criterion validity and moderate objectivity of the KIM-MHO draft 2007. The method was modified and evaluated again. It is planned to release a new version of KIM-MHO in spring 2012.
\end{abstract}

Keywords: key indicator method, manual handling operations, risk assessment, repetitive work tasks, hand arm system, Upper extremity musculoskeletal disorders, UEMSDs

\section{Introduction}

Upper extremity musculoskeletal disorders (UEMSDs) are still common in the working population [1]. In addition to computer work, heavy loads, high forces, awkward postures, and repetitive movements are the most frequently discussed work-related physical factors $[2,3]$.

According to the European Council Directive $89 / 391 /$ EEC of 12th June 1989 on the introduction of measures to encourage improvements in the safety and health of workers at work, the employer must perform an assessment of the risks to safety and health at work, including those to which specific groups of workers are exposed [4].
Long-time, dynamic repetitive exposure of the hand-arm system during manual handling operations (MHO) alone or in combination with static and postural effort are recognised as causes of musculoskeletal symptoms and disorders. The assessment of these manual work tasks is crucial to estimate health risks of exposed employees. For these work tasks, a new method for the assessment of the working conditions was developed by the Federal Institute for Occupational Safety and Health (BAuA) and released as a draft in the year 2007 [5]. The draft of the so-called Key Indicator Method for Manual Handling Operations (KIM-MHO) was developed in analogy with the existing KIM for Lifting/Holding/Carrying (KIMLHC) and Pulling/Pushing (KIM-PP) of loads [6].

*Corresponding author. E-mail: klussmann@institut-aser.de 
The KIM-MHO was designed to fill the gap existing in risk assessment of manual work processes, since the existing KIMs deal only with manual handling of loads.

The key indicators considered in the KIM-MHO are:

(1) daily duration of manual work processes,

(2) type, duration, and frequency of executing forces,

(3) body posture during manual work processes,

(4) hand-arm posture during manual work processes,

(5) work organisation and

(6) work conditions.

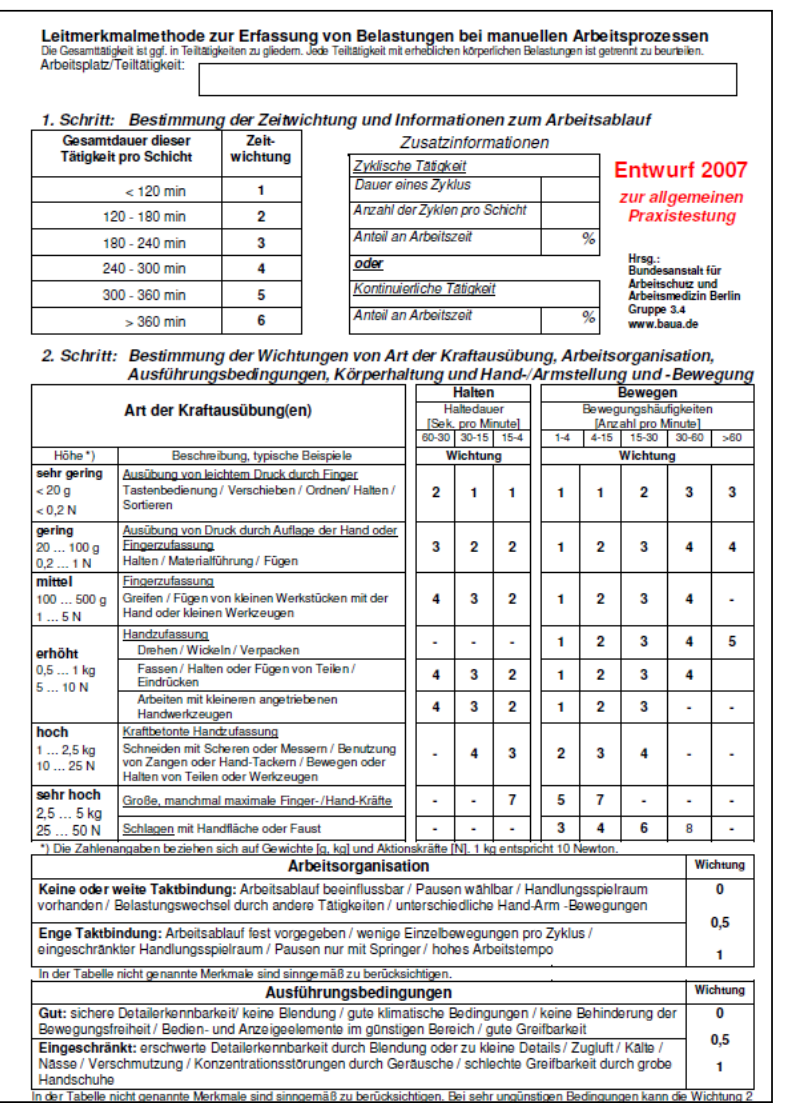

The key indicators are classified in different scales. By multiplying the scale value of the daily duration of the activity (1) with the sum of the other scale scores (2 to 6$)$, a total value can be calculated. This score can be allocated to a risk range: low exposure situation where physical overload is unlikely to occur ( $<10$ points), situations with increased $(10-<25$ points) and highly increased ( $25-<50$ points) exposure, up to conditions where physical overload is highly likely to occur with probable necessity to redesign the workplace ( $>=50$ points), see figure 1 .

Figure 1: Key-Indicator-Method “Manual Handling Operations” (KIM-MHO), draft 2007 [5].

\section{Methods and Design of the Study}

\subsection{Aim of this research project}

The aim of this research project was to evaluate the draft of the KIM-MHO [5]. The objectivity and

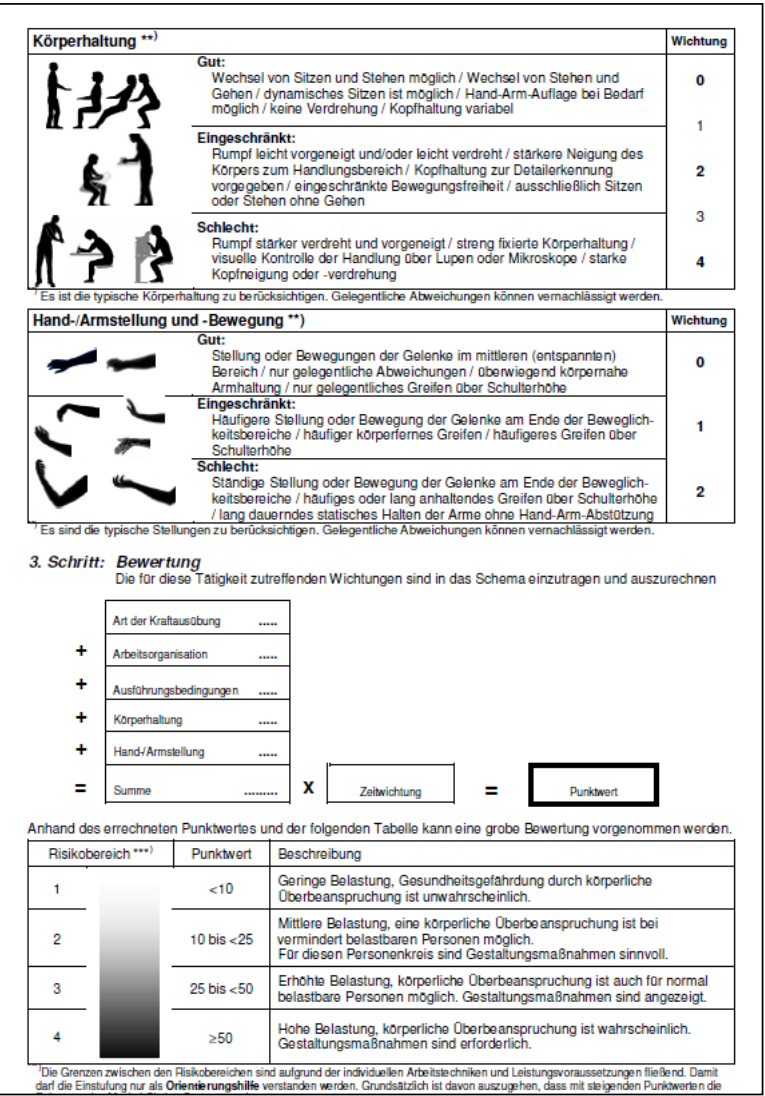


quency of musculoskeletal symptoms within the exposed workers (criterion validity). The study was designed as a cross-sectional study in Germany.

\subsection{Research topics}

The objectives above suggest the following working hypotheses:

\subsubsection{Research topic 1: objectivity and reliability}

Assessing workplaces by means of scientists experienced in ergonomics and by means of occupational health and safety officers using the KIM-MHO, will result in no significant discrepancies. Furthermore, no significant discrepancies occur between the assessments within the different occupational health and safety officers involved.

\subsubsection{Research topic 2: criterion validity}

It is assumed, that employees at workplaces with high exposures of manual handling operations show health related outcomes (musculoskeletal symptoms) more frequently than non-exposed workers, taking into consideration relevant confounders such as age, gender, constitution or disposition. Secondly it is assumed, that the KIM-MHO shows high scores at workplaces with high degrees of manual handling operations and high frequencies of musculoskeletal symptoms in exposed workers, and will result in low scores at workplaces with low exposures of manual handling operations and low frequency of musculoskeletal symptoms in workers.

\subsection{Instruments}

The instruments (i.e. standardised questionnaire, assessment of exposure) used in this survey were used in a similar form in other studies by several authors. Among them, the authors of the present study applied them for the assessment of musculoskeletal symptoms of upper extremities and the neck in office workers [3]. This former study sample was used as a reference data set.

\subsubsection{Assessment of health outcome}

A survey among exposed employees was carried out using a standardised questionnaire. The questionnaire was based predominantly on the so called Nordic Questionnaire [7].

\subsubsection{Assessment of exposure}

The working conditions of the employees were documented by ergonomic work procedure analysis and time analysis including task observation, time measurements, and assessment of technical procedures.

\subsubsection{Application of the KIM-MHO}

Application of the KIM-MHO based on the exposure assessment.

To determine the objectivity and reliability of workplace assessment, occupational health and safety officers at different workplaces involving manual handling operations applied the KIM-MHO. In order to determine the criterion validity of risk assessment, a survey of employees at different workplaces took place with standardised questionnaires and interviews about symptoms in the neck and upper extremities.

\section{Results}

\subsection{Analysis of research topic 1}

Whithin cross sectional studies, 17 different work places with manual handling operations were included. At every workplace, an extensive work analysis was carried out to gather relevant data about respective manual handling operations (duration of tasks, frequency, force, posture, etc.). Relevant work tasks were documented by video analysis. From this data, 6 workplaces were selected and the KIM-MHO was used by scientists and occupational health and safety officers separately to assess the working conditions. The difference between the real exposure (as assessed by extensive work analysis) and the assessment by KIM-MHO were used to describe the objectivity and reliability of the KIM-MHO. 56 participants (occupational health and safety officers) could be recruited for this study. Three groups (A, B and C) were formed (see table 1) and each group assessed two work places with the KIM-MHO (=6 different workplaces).

Descriptive statistics were used to show the distribution of different workplace assessments of the involved experts (see figure 2). 
Table 1

Description of the participants (occupational health and safety officers) during the analysis of objectivity and reliability of KIM-MHO, draft 2007

\begin{tabular}{|c|c|c|c|c|}
\hline & Group A & Group B & Group C \\
\hline \multicolumn{2}{|c|}{$\begin{array}{l}\text { number of } \\
\text { participants }\end{array}$} & 23 & 15 & 18 \\
\hline \multirow{4}{*}{$\begin{array}{l}\text { age } \\
\text { group }\end{array}$} & $<30$ years & 2 & 1 & 0 \\
\hline & $30-<40$ years & 6 & 4 & 3 \\
\hline & $40-<50$ years & 7 & 4 & 6 \\
\hline & $>50$ years & 7 & 5 & 8 \\
\hline
\end{tabular}

The correlation between the work place assessments (using the KIM-MHO) within the occupational health and safety officers and in comparison to scien- tist experienced in ergonomics was moderate to good. The difference between the assessment of the scientist and the median value of the health and safety officers was nearly equal in all assessments. However, partially some officers differed clearly from the median value.

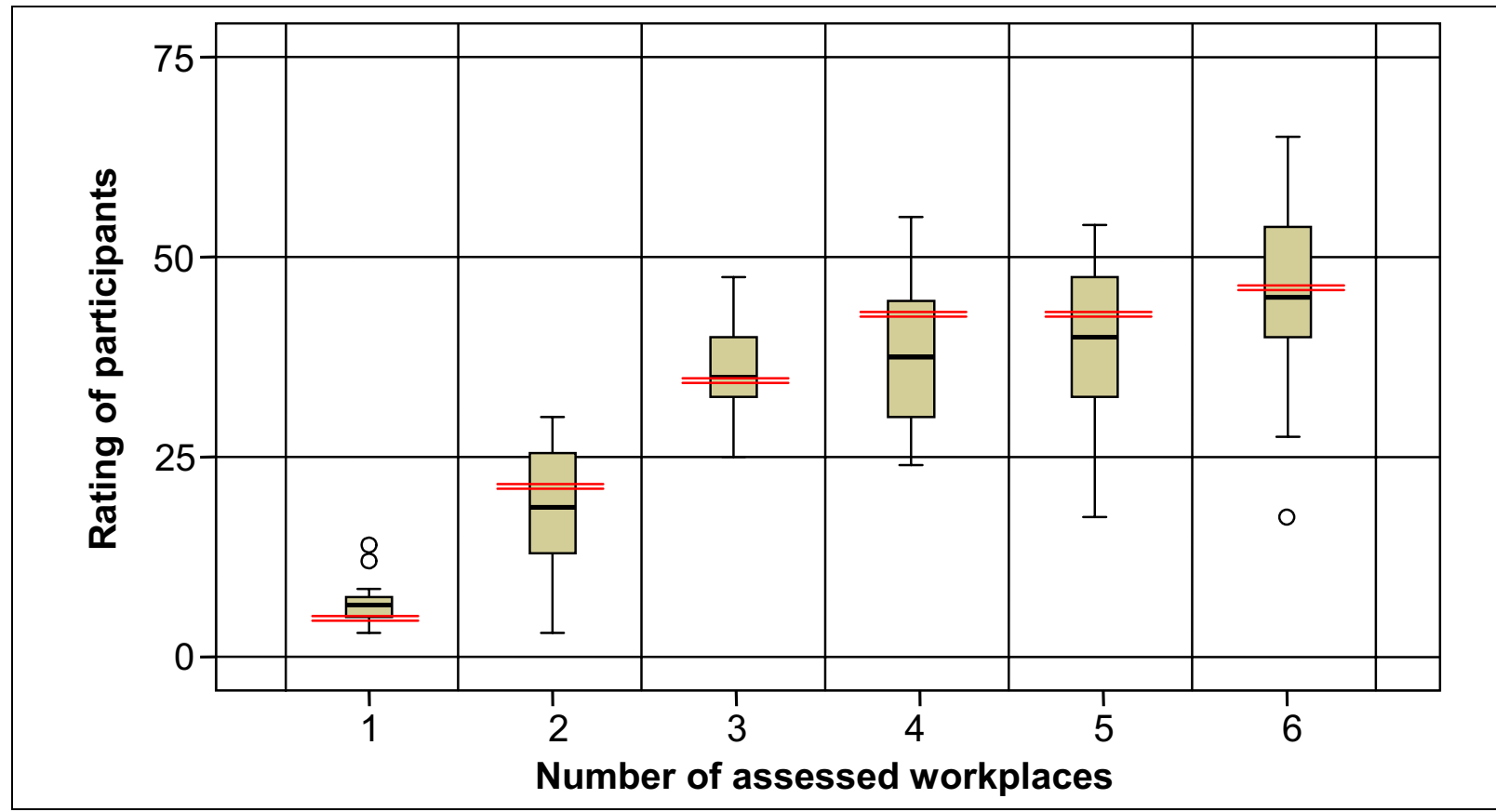

Figure 2: Assessment of 6 work places with the KIM-MHO by scientist experienced in ergonomics (double lines) and 56 occupational health and safety officers (divided in 3 groups, each group assessed two workplaces). The distribution of the assessment of the occupational health and safety officers is shown in box plots, workplaces are sorted by rating of the ergonomic experts.

\subsection{Analysis of research topic 2}

It is assumed, that employees at workplaces with high exposures of manual handling operations show health related outcomes (musculoskeletal symptoms) more frequently than non-exposed workers, taking into consideration relevant confounders such as age, gender, constitution or disposition. Secondly it is assumed, that the KIM-MHO results in high scores at workplaces with high degrees of manual handling operations and high frequencies of musculoskeletal symptoms in exposed workers, and low scores at workplaces with low exposures of manual handling operations and low frequencies of musculoskeletal symptoms in workers.

Whithin cross sectional studies among 17 different work places, a total number of 642 employees (207 women, 435 men) with exposures of manual handling operations were included. 
In order to estimate whether prevalences of symptoms in the upper extremities and neck were excessive, the data from the employees of each workplace were compared with a similar reference data set among employees working at visual display terminal (VDT) workstations. These reference data were generated in a cross-sectional study of 804 employees working at VDT [5].

The work places were analysed by the KIM-MHO and the prevalence (12-month prevalence) of symptoms in the musculoskeletal system was documented. The distribution of the employees assigned to "risk categories" (determined by the
LMM-MHO, draft 2007) related to the manual work processes is described in table 2 .

Table 2

Distribution of the employees assigned to "risk categories" (determined with the LMM-MHO, draft 2007) related to the manual work processes

\begin{tabular}{|c|c|c|c|c|}
\hline & & \multicolumn{2}{|c|}{ gender } & \\
\hline $\begin{array}{c}\text { risk } \\
\text { category }\end{array}$ & value & male & female & all \\
\hline 1 & $<10$ & 498 & 306 & 804 \\
\hline 2 & 10 bis $<25$ & 172 & 70 & 242 \\
\hline 3 & 25 bis $<50$ & 76 & 73 & 149 \\
\hline 4 & $>50$ & 61 & 190 & 251 \\
\hline
\end{tabular}

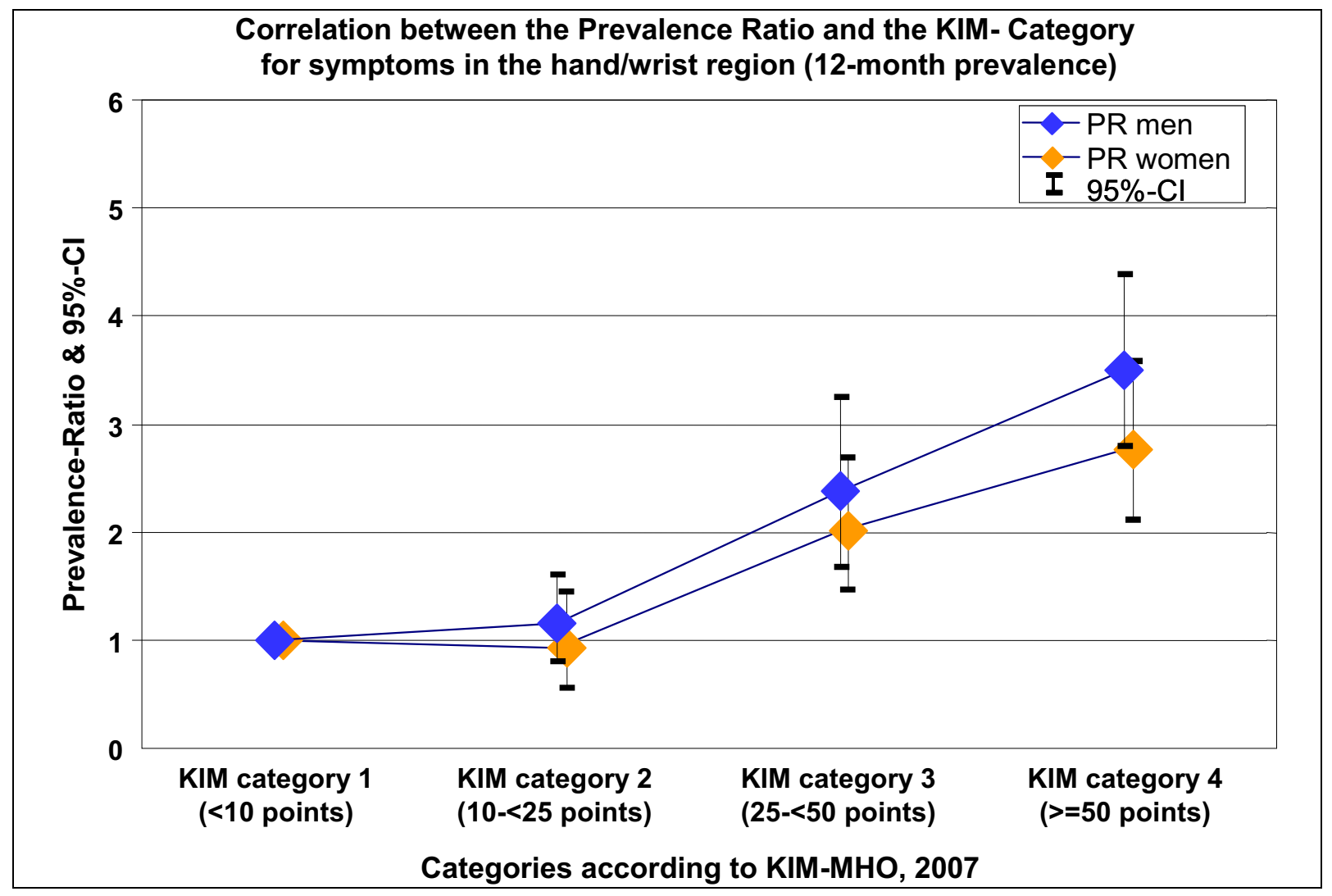

Figure 3: Prevalence Ratios of musculoskeletal symptoms in correlation with the risk categories of the KIM-MHO (general linear model: logbinomial, adjusted for age, height and BMI, stratified for gender, $95 \%$ confidence intervals $(95 \%$-CI)).

Multivariate regression analyses based on logbinomial models were used for multivariate comparisons between exposed and unexposed subjects. Prevalence Ratios were calculated as effect estimates. Relevant confounders (age, constitution, disposition, behaviour, work history) were taken into consideration. Data were generally stratified by gender. Considering the Prevalence Ratios of musculoskeletal symptoms in the hand/wrist region (figure 3) and elbow/forearm region (figure 4) in correlation with 


\section{Table 3}

the risk categories of the KIM-MHO, a clear dose response relationship is visible. The Dose-ResponseRelationship between the KIM-MHO-score and symptoms in all considered body region are summarised in table 3 .
Dose-Response-Relationship between the KIM-MHO-score and symptoms in the respective body region

\begin{tabular}{|l|c|c|}
\hline body region & male & female \\
\hline hand/wrist region & +++ & +++ \\
\hline elbow/forearm region & ++ & +++ \\
\hline shoulder region & + & + \\
\hline neck region & - & - \\
\hline
\end{tabular}

The results of the evaluation of the criterion validity support a dose-response relationship between the amount of exposition to manual handling operations (KIM categories with $25-50$ points and $>50$ points) and symptoms in hand/wrist region, elbow/forearm region and shoulder region but not to neck region.

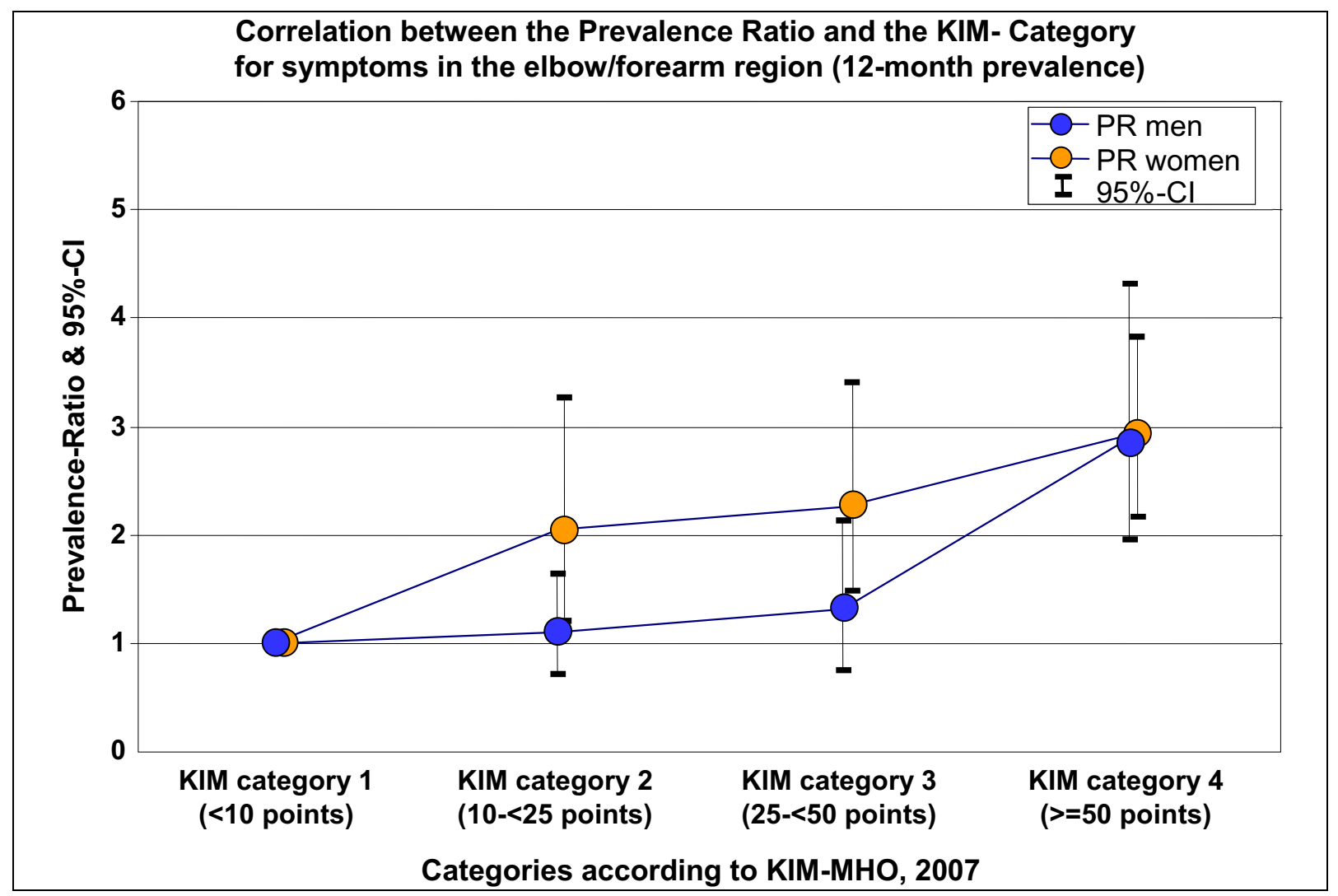

Figure 4: Prevalence Ratios of musculoskeletal symptoms in correlation with the risk categories of the KIM-MHO (general linear model: logbinomial, adjusted for age, height and BMI, stratified for gender, $95 \%$ confidence intervals (95\%-CI)).

\section{Discussion}

The results suggest satisfying criterion validity and moderate to good objectivity and reliability of the KIM-MHO draft 2007. Areas for modification to improve this method for assessment of the health risk of manual handling operations at diverse workplaces are indicated.

At present, a new version of the KIM-MHO is developed, considering the findings from this study and additional advices from practical experiences collected from practical users in the years since first draft of KIM-MHO has been published. 
For the estimation of the criterion validity, the same database of musculoskeletal disorders of the employees and the workplace analysis can be used. To assess objectivity and reliability, an analysis with the new version of the method is required.

It is planned to release a new version of KIMMHO in spring 2012.

\section{References}

[1] Roquelaure Y, Ha C, Leclerc A, Touranchet A, Sauteron M, Melchior M, Imbernon E, Goldberg M: Epidemiologic surveillance of upper-extremity musculoskeletal disorders in the working population. Arthritis Rheum 2006, 55(5):765-78.

[2] Viikari-Juntura E, Martikainen R, Luukkonen R, Mutanen P, Takala EP, Riihimaki H: Longitudinal study on work related and individual risk factors affecting radiating neck pain. Occup Environ Med 2001, 58(5):345-352.
[3] Klussmann A, Gebhardt H, Liebers F, Rieger MA: Musculoskeletal symptoms of the upper extremities and the neck: a cross-sectional study on prevalence and symptom-predicting factors at visual display terminal (VDT) workstations. BMC Musculoskelet Disord 2008, 9:96.

[4] Council Directive 89/391/EEC of 12 June 1989 on the introduction of measures to encourage improvements in the safety and health of workers at work [http:/ / eur-lex.europa.eu/ LexUriServ/ LexUriServ.do? uri=CELEX:31989L0391: EN:HTML]

[5] Steinberg U, Behrendt S, Caffier G, Schultz K, Jakob M: Leitmerkmalmethode Manuelle Arbeitsprozesse. Forschung Projekt F 1994. Berlin: BAuA Eigenverlag; 2007.

[6] SLIC European inspection and communication campaign: LIGHTEN THE LOAD.

http://www.handlingloads.eu/en/site/18/19

[7] Kuorinka I, Jonsson B, Kilbom A, Vinterberg H, BieringSorensen F, Andersson G, Jorgensen K: Standardised Nordic questionnaires for the analysis of musculoskeletal symptoms. Appl Ergon 1987, 18(3):233-237. 